Service social

\title{
Pratiques interculturelles sous l'angle de la modernité, par Ghislaine Roy, Montréal, Centre de services sociaux du Montréal métropolitain, 1991.
}

\section{François Larose}

Volume 42, numéro 1, 1993

Culture et intervention

URI : https://id.erudit.org/iderudit/706607ar

DOI : https://doi.org/10.7202/706607ar

Aller au sommaire du numéro

Éditeur(s)

École de service social de l'Université Laval

ISSN

1708-1734 (numérique)

Découvrir la revue

Citer ce compte rendu

Larose, F. (1993). Compte rendu de [Pratiques interculturelles sous l'angle de la modernité, par Ghislaine Roy, Montréal, Centre de services sociaux du Montréal métropolitain, 1991.] Service social, 42(1), 157-159.

https://doi.org/10.7202/706607ar d'utilisation que vous pouvez consulter en ligne.

https://apropos.erudit.org/fr/usagers/politique-dutilisation/ 
produit à l'intérieur du circuit collégial. II existe pourtant des définitions opératoires du stéréotype qui sont, d'emblée, mises en relation avec le concept de «représentation sociale» dont le stéréotype* est le plus souvent un élément structurant. De la même façon, pourquoi nous présenter une définition à la fois partielle et douteuse du concept d'acculturation produite par un glorieux inconnu, alors que la littérature scientifique en anthropologie et en psychologie renvoie généralement à une définition originale produite par Herskovits (1938)?

Dans l'ensemble, il s'agit donc d'un document d'information intéressant qui ne saurait, cependant, être considéré d'aucune façon comme un document de formation.

FrançOis LAROSE

Département d'enseignement au préscolaire et au primaire Université de Sherbrooke

\section{PRATIQUES INTERCULTURELLES SOUS L'ANGLE DE LA MODERNITÉ}

Ghislaine Roy,

Montréal, Centre de services sociaux du Montréal métropolitain, 1991.

Ce document, édité par le CSSMM, est en fait la reproduction d'un mémoire de maîtrise en service social rédigé par une intervenante chevronnée du réseau des services sociaux. L'auteure y définit en premier lieu son cadre théorique. Après avoir situé la pluriethnicité québécoise dans le contexte historique de I'accès à la "modernité », I'auteure aborde rapidement le concept du multiculturalisme en tant que discours idéologiquement marqué. Elle situe ensuite le développement des réseaux formels ainsi que des pratiques des services sociaux québécois à l'intérieur d'une même perspective dynamique d'accès à la modernité. Elle situe notamment, de façon fort judicieuse, la notion de parallélisme des services, des réseaux et des infrastructures entre les deux communautés culturelles dont le statut de pérennité est reconnu: les communautés franco-québécoise et angloquébécoise.

Si la notion de modernité est à la fois bien définie sur le plan théorique et clairement articulée dans une perspective socio-historique, certains construits complexes sont définis quelque peu rapidement et sommairement. Il en est ainsi du construit de culture ou de I'utilisation dans une

* Voir à ce propos: J.-P. Leyens, Sommes-nous tous des psychologues? Bruxelles : Mardaga, 1983. 
acception peu orthodoxe du concept de socialisation ou encore de la définition informelle et incorrecte du construit de représentation sociale. De la même façon, l'auteure énonce plusieurs descriptions de modèles $d^{\prime}$ intervention en travail social auprès de clientèles d'origines ethniques et culturelles minoritaires. Si ces descriptions sont intéressantes, on ne parvient guère à en dégager un modèle dominant ou privilégié par l'auteure, mis à part la reconnaissance que la majeure partie des auteurs des ouvrages qu'elle a consultés reconnaissent l'existence de différences entre cultures occidentales et non occidentales. Ces différences devraient être prises en considération dans l'analyse des rapports entre l'appareil (les services sociaux) et le client (le migrant).

En fait, la plus grande faiblesse de cette partie de l'ouvrage réside probablement dans l'absence d'une modélisation des processus d'acculturation et des impacts de ces derniers à la fois sur le plan individuel et collectif. Cette carence semble se fonder sur une méconnaissance générale des travaux produits depuis quarante ans en psychologie interculturelle ainsi que de ceux produits dans le domaine des culture-bound syndromes en psychiatrie. II est regrettable que l'auteure n'ait pas tenu compte, notamment, des travaux de Berry* sur le stress acculturatif. Ces travaux forment le modèle de référence utilisé par l'OMS en la matière. Les travaux de Berry, Jahoda, Lonner, Draguns, Triandis et autres psychologues anglo-saxons se retrouvent en toile de fond, notamment, des textes de Mme Cohen-Emerique auxquels I'auteure se réfère souvent.

La seconde partie de l'ouvrage est consacrée au cadre méthodologique. L'auteure nous y présente une approche intéressante alliant à la fois l'analyse de contenu du discours d'intervenantes et d'intervenants du réseau des affaires sociales de la région de Montréal à des incidents survenus dans leur pratique auprès de migrantes et de migrants et l'analyse du discours des mêmes intervenantes et intervenants à leur perception d'une pratique interculturelle. Le fait que le document soit relativement faible sur le plan de la méthodologie et qu'on y demeure à un niveau fort primaire de la justification de l'approche choisie par l'opposition mécaniste entre approche qualitative et analyse de données quantitatives se trouve appuyé par le caractère exploratoire et descriptif de la recherche présentée.

La troisième partie porte sur le traitement des données recueillies auprès de quatorze intervenantes et intervenants du réseau des affaires sociales de la région montréalaise. L'auteure y fait ressortir ce qui, d'après elle, constitue des incidents mettant en cause une différence interculturelle entre les critères psychosociaux des praticiennes et des praticiens sociaux et ceux de leurs clientèles. L'auteure regroupe ces « incidents » en quatre grandes sous-caté-

J.W Berry et V. Kim, "Acculturation and Mental Health», dans P.R. Dasen, J.W. Berry et N. Sartorius (ed.) Health and Cross-cultural Psychology, Toward Applications, Beverly Hills: Sage, p. 207-238. 
gories conceptuelles représentant les principales zones de conflictualités interculturelles entre migrants et intervenants des réseaux des sociétés occidentales. Bien qu'intéressante, la catégorisation basée sur un modèle bâti par une psychologue française, Margalit Cohen-Emerique, renvoie à des construits qui sont $d^{\prime}$ 'une ampleur et d'une complexité fort variables. En conséquence, ces concepts sont plus ou moins opérationnalisables, ce qui affecte directement le traitement que l'auteure fait des informations qui lui sont fournies par les praticiennes et les praticiens auxquelles elle se réfère. En fait, tout au long de ce qui est décrit comme des produits de la différence entre la culture du réseau et celles des clients, on ne sait trop si l'interprétation porte sur une réaction de l'intervenante ou de l'intervenant par rapport à une dimension culturelle ou s'il s'agit d'une réaction à l'évaluation d'un problème d'ordre socio-économique.

La sous-section suivante nous présente le résultat d'une brève analyse de contenu des déclarations des praticiennes et praticiens relativement à ce qu'elles ou ils définissent comme les déterminants de leurs pratiques interculturelles. Bien qu'elle soit quelque peu sommaire du point de vue de I'exploitation des données, cette sous-section fournit un éclairage fort intéressant des perceptions des intervenantes et des intervenants sur leur propre pratique. Par cette analyse de contenu, I'auteure fait ressortir des communautés de thèmes entre les praticiennes et les praticiens tant en ce qui à trait à la définition d'une intervention interculturelle que par rapport à ce qui la distingue d'une pratique « régulière ». Elle y fait ressortir d'une façon originale l'importance du terrain pour ces intervenantes et intervenants ainsi que la nécessité d'un engagement subjectif et d'une attitude «quasi artisanale » de leur part. Si, en général, l'ouvrage porte souvent à la généralisation abusive ainsi qu'à l'interpénétration une peu trop étroite de la description et de I'interprétation subjective de l'auteure, la seconde partie du troisième chapitre (p. 59 à 76) est d'une richesse suffisante, sur le plan descriptif, pour en justifier la consultation.

On ne peut certes considérer ce document comme étant d'une grande portée scientifique. Le lecteur devrait tenir compte d'importantes limites tant sur le plan de l'exploration de la littérature scientifique en matière de psychologie ou de sociologie interculturelle que sur celui de la méthode adoptée. Néanmoins, il s'agit d'un ouvrage qui mérite d'être parcouru, ne serait-ce qu'en tant que reflet des préoccupations de praticiennes et de praticiens faisant face à des situations «a-normatives ». Il mérite aussi d'être diffusé en tant qu'instrument de sensibilisation à l'importance des dimensions subjectives dans le traitement de la relation praticien-clientèle dans un contexte socio-économique en pleine mouvance. 